Mostafa Alimehr ${ }^{1}$, Samira Malayen², Zahra Karimipour ${ }^{3}$, Kobra Doostifar ${ }^{4}$, Fereshteh Sohrabi Vafa ${ }^{5}$, Maryam Nikbina ${ }^{6}$

${ }^{1}$ Department of Health Services Management, Dezful University of Medical Sciences, Dezful, Iran

${ }^{2}$ Department of Midwifery, Dezful University of Medical Sciences, Dezful, Iran

${ }^{3}$ Department of Health Education, Dezful University of Medical Sciences, Dezful, Iran

${ }^{4}$ Department of Public Health, Shoushtar Faculty of Medical Sciences, Shoushtar, Iran

${ }^{5}$ Department of Community Medicine, School of Medicine, Dezful University of Medical Sciences, Dezful, Iran

${ }^{6}$ Department of Nursing and Midwifery, Shoushtar faculty of Medical Sciences, Shoushtar, Iran

\title{
Pre-diabetic women's understanding of the role of diet in preventing diabetes using the PEN-3 cultural model
}

\section{ABSTRACT}

Background. Pre-diabetes is one of the most widespread epidemics in the world. Pre-diabetics are the most susceptible to diabetes. The prevalence of prediabetes status is higher than that of diabetes. Lifestyle modification at this stage may delay the onset of type 2 diabetes for ten years. The present study aims at determining the Dezful pre-diabetic women's experiences of diabetic preventive eating behaviors.

Methods. The present study employed a directed qualitative content analysis (DQICA) conducted in Dezful in 2020. The experiences of 41 pre-diabetic women aged 30-65 years and 9 health care providers (physicians, nutritionists, public health experts) were explained using the in-depth interviewing technique on the factors affecting diabetes preventive behaviors, including nutritional behaviors and physical activities, based on the constructions of the PEN-3 model. The participants were selected from Dezful health centers. Analysis of the qualitative data was done via a directed qualitative content analysis (DQICA). Data validity was estimated by assessing acceptability, reliability, adaptability, and transferability.

Results: the most appropriate and accessible way to promote diabetes prevention behaviors in pre-diabet-

Address for correspondence:

Fereshteh Sohrabi Vafa

Department of Community Medicine, School of Medicine

Dezful University of Medical Sciences, Dezful, Iran

e-mail: fereshteh.sohrabi1399@gmail.com

Clinical Diabetology 2021, 10; 3: 261-269

DOI: 10.5603/DK.a2021.0023

Received: 06.10.2020

Accepted: 28.01.2021 ics is to hold training classes with a health promotion approach implemented by health care providers. Conclusion. Pre-diabetic women have a variety of beliefs and habits influenced by awareness, food culture, and temporal and spatial constraints. By identifying the positive and negative beliefs affecting diet, researchers who want to promote the nutritional behavior of pre-diabetic women should correct and eliminate negative and inhibitory beliefs by targeting these beliefs, emphasizing positive and motivating beliefs. They also should use the mentioned points in their educational interventions. (Clin Diabetol 2021; 10; 3: 261-269)

Key words: PEN-3 cultural model, pre-diabetic, education, perceptual factors, enablers

\section{Introduction}

In the $21^{\text {st }}$ century, chronic diseases account for more than 35 million people annually or nearly twothirds of all deaths worldwide [1]. According to the World Health Organization (WHO), 60\% of the causes of death and $43 \%$ of the global burden of disease (GBD) are related to non-communicable diseases, of which diabetes is one of the most common chronic diseases worldwide [2]. Diabetes, as a chronic disease, is significantly involved in the spread of physical injury, mortality, and health costs [3]. It is an increasingly important public health concern that is considered as one of the most important health, medical and socio-economic problems in the world today [2] so that the WHO calls it a silent epidemic [4].The disease is influenced by environmental and hereditary factors. Also, a sedentary 
lifestyle is associated with an increased risk of obesity, increased waist circumference, insulin resistance, and cardiovascular disease. Ultimately, it causes a decrease in the quality of life. If the prevention and control strategies of diabetes are not implemented, the number of diabetic patients will increase day by day [5].

The prevalence of pre-diabetes and diabetes has been on the rise in recent years, with the WHO estimating that about 422 million people worldwide have diabetes, most of them living in low- and middle-income countries. Diabetes also causes 1.6 million deaths annually [6]. Prediabetes is a complication in which the fasting blood sugar level is higher than normal, but this increase is not enough to be considered diabetes [5]. Studies have shown that modifying the pre-diabetes stage can reduce the chances of developing type 2 diabetes in pre-diabetic people by up to $60 \%$. Also, by changing the lifestyle, $90 \%$ of type 2 diabetes can be prevented $[5,6]$.

It is possible to prevent pre-diabetes to type 2 diabetes by using a healthy diet, losing weight, maintaining a healthy weight, and doing exercises [7]. Therefore, for people to learn the right lifestyle to maintain health and prevent disease, they need to change unhealthy behaviors to healthy behaviors and implement educational programs to achieve these behaviors [8]. Researchers have proposed various theories and models to explain the determinants and factors affecting behavior. One of these models is the PEN-3 model, in which culture is the axis and basis in preventive behavior or health-promoting behavior [9]. Culture is an important underlying factor that shapes human behavior, and should be considered in all health promotion programs aimed at improving and modifying individuals' habits. Regarding diabetes self-care process, in which individuals' health behaviors shape the context of the family, community and society, all cultural factors play positive and negative roles [10].

The PEN-3 model is one of the health education models used to report a collection of health subjects (e.g., cancer, HIV, diabetes, nutrition) with compound clinical and cultural backgrounds affecting health behaviors and health outcomes [11].

The PEN-3 Cultural Model identifies 3 domains of focus, and each domain includes three factors that form the acronym PEN (Figure 1):

1. Cultural identity identifies the persons, extended family, and neighborhoods that should be addressed in health education. Individuals within each category should be educated and empowered to make informed health decisions appropriate to their roles in the family and community.

2. Relationships and expectations explores the intended audience's perceptions of health infor-

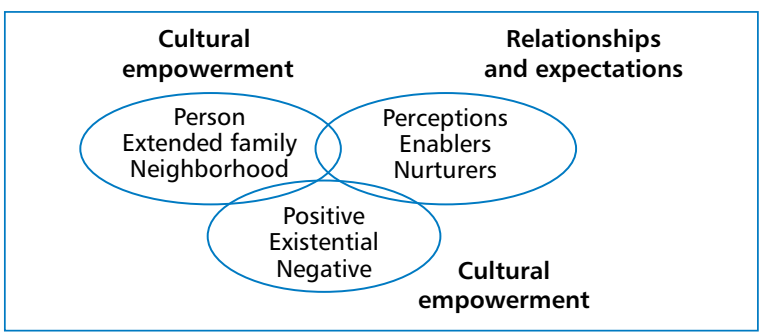

Figure 1. PEN-3 model

mation and the factors that enable and nurture health behaviors.

3. Cultural empowerment encompasses the positive, existential and negative dimensions of a person's culture that can be used to empower individuals to adopt healthy behaviors [12].

Given that the foundations of healthy and preventive behaviors are laid within society, as a result, women's culture, beliefs, and ideas have a great effect on the acceptance of a healthy diet. For this purpose, it is necessary to use a qualitative approach to explain the cultural and social factors affecting the adoption and also to perform nutritional behaviors to prevent diabetes among women, which can be a way to prevent type 2 diabetes among women. The present study aims to explain the Dezful pre-diabetic women's experiences of diabetic preventive nutritional behaviors based on the PEN-3 cultural model constructs including perceptual factors, enablers, and reinforcers in three positive, negative, and neutral dimensions.

\section{Methodology}

The present study employed a directed qualitative content analysis (DQICA) conducted in 2020 in Dezful city. The research population consisted of all pre-diabetic women referred to health centers, physicians' offices, and members of the Dezful city health care team. Inclusion criteria include: being in the age range 30-65 years (age of diabetes screening), having the willingness to participate in the study, having FBS level 100-125 mg/dl, HbA1c 5.7-6.4\%, and having a family record in the health centers. According to the aim of the study, the participants were initially selected via a purposive sampling method with maximum diversity in terms of age, level of education, occupation, ethnicity, etc. from among pre-diabetic women aged 30 to 65 years, who were referred to four health centers under the auspices of the health center affiliated to Dezful university of medical sciences. The participants were 41 pre-diabetic women with a mean age of 44.85 \pm 9.80 who did not take blood glucose control drugs and could communicate properly and provide rich and 
complete information to the researchers. Their names were based on the result of a free blood glucose test (FBS, $\mathrm{HbA}_{1 \mathrm{c}}$ ) was recorded by laboratory personnel. They also had previously been advised by health center staff to change their lifestyle.

The participants were excluded from the study if they were dissatisfied or had symptoms of diabetes, such as a heart attack or stroke. Pregnant and lactating women were not interviewed and were excluded from the study as well. In the PEN-3 model, enablers were individuals outside the population at risk who control or influence environmental conditions to improve the health of individuals at risk. These factors include, for example, family members, policymakers, legislators, resource controllers, or health care providers [13].

In this study, physicians and members of the health care team played a significant role as environmental factors in controlling blood sugar levels in pre-diabetic women. Therefore, in addition to pre-diabetic women, according to the research aim, in-person interviews were conducted with the staff of the health center. A total of 41 women and 9 health staff were interviewed. After the necessary coordination and personal follow-up, the participants attended the interview sessions. The number of sessions and the duration of each interview varied according to the content of the subject and the conditions of each participant. Interviews with prediabetic women were conducted in neighborhoods at the suggestion of the participants, who usually chose a place where they could feel comfortable and express their experiences. These neighborhoods included health centers. At the beginning of an interview, the objectives of the study, methods, and steps of interviewing women were discussed and the participants were assured that the information would be used only for research and no name or address would be asked or recorded anywhere. After their consent, semi-structured interviews were conducted, and with the permission of the participants, while briefly taking notes of non-verbal observations and behaviors, the interviews were recorded and analyzed on the same day. The interview begins with an open and general question to clarify the subject of the interview. In conducting the interviews, an attempt was made to minimize interference in the interview process, and at the same time, the deviation of the interview path was prevented with appropriate questions. Since in this study the guided content analysis method has been used, the framework for guiding interview questions was set based on the structures of the PEN-3 model through specialized consultation with professors in the field of health education and health promotion. Interviews and data analysis continued until saturation. Each interview lasted approximately 20 to 60 minutes.
First, general questions were asked to start the interview and the interview process was guided based on the participants' answers. The interviewer listened carefully to the interviews several times and reviewed the manuscript text several times. At the end of each interview session, the data recorded by the researcher were heard at the earliest opportunity and compared with the note-taking notes, and the contents were merged and corrected. The text was then read several times to gain a deep and accurate understanding of it. It was reduced to the smallest meaningful units (codes). The codes were then classified according to the constructs of the PEN-3 model (Table 1). In this research, the data were analyzed using the qualitative content analysis method, and according to Granheim and Landman's (2004) approach [14].

Lincoln \& Guba's (1985) trustworthiness criteria and techniques including credibility, confirm ability, dependability, and transferability were used to evaluate the accuracy and precision of qualitative data [15].

\section{Results}

Description of themes and (positive, negative, and neutral) categories

\section{Perceptual factors}

Refer to beliefs and convictions that promote or hinder health behavior or have no effect on health performance and are divided into three subcategories of positive perceptions, negative perceptions, and neutral perceptions.

1. Positive perceptions. It is the first category in the data and refers to perceptions and beliefs that help promote health behavior. Positive perceptions included 4 subclasses expressed by pre-diabetic women.

A. Knowledge of the cause of pre-diabetes. In addition to being pre-diabetic, most participants had other conditions, such as high blood lipidogram, high blood pressure, and obesity, which they blamed on a poor diet for their medical problems. One pre-diabetic woman with high blood lipidogram said: "I know now that my obesity and blood sugar level is higher than the normal, and it is because I do not follow my diet at all" (Interviewee \#2).

B. Belief in achieving physical health with healthy nutrition. The pre-diabetic women understood the benefits of dieting and its importance in controlling the disease: "I have been reducing my sugar intake for a month with the explanations given to me by the health care center's experts" (Interviewee \#11).

C. Belief in preventing diabetes and its complications with a healthy diet. "Patients with a family record of diabetes and who have had diabetes and 
Table 1. The process of analyzing qualitative nutrition data

\begin{tabular}{|c|c|c|}
\hline Themes & Categories & Sub-categories \\
\hline \multirow[t]{12}{*}{ Perceptions } & Positive perceptions & Awareness of the cause of prediabetes (poor diet, inheritance) \\
\hline & & $\begin{array}{l}\text { Belief in achieving physical health with healthy nutrition (controlling blood sugar levels, } \\
\text { weight loss) }\end{array}$ \\
\hline & & Belief in preventing diabetes and its complications with a healthy diet \\
\hline & & Belief in reducing the cost of treatment with a healthy diet \\
\hline & Negative perceptions & Lack of adequate knowledge of healthy foods \\
\hline & & Insufficient knowledge of how to properly consume food \\
\hline & & $\begin{array}{l}\text { Lack of knowledge about how to cook food properly (for example, how to cook traditional } \\
\text { food healthier) }\end{array}$ \\
\hline & & Belief in diabetes only by eating sugary foods \\
\hline & & Mental problems preventing diet (belief in uncontrollable appetite) \\
\hline & & Interest in eating ready-made and modern foods \\
\hline & Existing perceptions & A greater tendency to follow a diet than to exercise \\
\hline & & Cooking methods based on taste and smell (spicy foods) \\
\hline \multirow[t]{11}{*}{ Enablers } & Positive enablers & Effective training of health care providers (physicians and experts) \\
\hline & & Easy access to the health care unit \\
\hline & & Media education (Radio \& television) \\
\hline & & Free services of health centers (diabetes screening plan - tests, etc.) \\
\hline & Negative enablers & Lack of counseling facilities in the health center (psychiatrist - nutritionist) \\
\hline & & Failure to file and follow up on pre-diabetics \\
\hline & & The insufficient income of families \\
\hline & & Inefficient referral system services \\
\hline & & Insufficient training (familiarity with healthy cooking methods, etc.) \\
\hline & Existing enablers & Belief in the use of herbal medicines (traditional medicine) in controlling blood sugar \\
\hline & & Preparation of food by working together \\
\hline \multirow[t]{19}{*}{ Reinforcers } & Positive reinforcers & Family members' (parents') encouragements \\
\hline & & Other family members' encouragements \\
\hline & & Friends' encouragements \\
\hline & & Physicians' encouragements \\
\hline & & Health staff's encouragements \\
\hline & & Good communication and attitude of the center staff \\
\hline & & Communication with individuals and families with diabetes \\
\hline & & Positive behavioral experiences of dieting \\
\hline & & Experience losing weight - lowering blood sugar levels — reducing symptoms \\
\hline & Negative reinforcers & High interest in consuming sugary foods and sweets \\
\hline & & The idea of separating your food from the family food in case of a certain diet \\
\hline & & Inability to follow a diet when eating with family members \\
\hline & & The family members' insistence on neglecting the disease \\
\hline & & The family members' mere advice to diet and their no accomplishment with the patient \\
\hline & & The tendency of family members, especially the youth (children), to consume fast food \\
\hline & & The patient's no power in selecting and preparing food \\
\hline & & Family lifestyle (low attention to self-care behaviors) \\
\hline & Existing reinforcers & Planning of food and preparation of a list with family members \\
\hline & & Preparation of a shopping list of raw materials needed to prepare food with family members \\
\hline
\end{tabular}

problems with them feel that they are at risk of diabetes or its complications. They are more likely to follow a diet and pay attention to prevention", said the center's expert.
D. Belief in reducing the cost of treatment with healthy nutrition. Some participants emphasized that dieting prevents diabetes and pays for expensive blood sugar-controlling medications, eliminating the need 
for a high-profile visit to a specialist and paying for a test. "If I become diabetic, I have to go to a specialist regularly and pay a lot of money for visits and medication" (Interviewee \#16).

2. Negative perceptions. The second category is in the data and refers to perceptions and beliefs that prevent health behavior and included 6 subcategories.

A. Lack of adequate knowledge of healthy foods. The staff at the health center said most of the patients" information was poor and often incorrect: "We eat a lot of beans in this region. Until now, I thought it was a vegetable. I ate a lot, but now I realize that some vegetables have starch like beans and I have to adhere to the diet" (Interviewee \#17).

B. Insufficient knowledge of how to eat properly. "Both pre-diabetics and diabetics do not know how much fiber should be in their daily diet. For example, what kind of fruit should they eat daily to meet their needs, so they all need training?" (the center's expert). C. Lack of knowledge of the proper cooking method. Lack of knowledge about how to change the way food is cooked, as well as lack of knowledge about the nutritional value of basic food ingredients, make them not have a healthy diet, which was mentioned by some women and staff of the center. "Samosa, as our traditional food, is mostly made of potatoes and fried in oil. No one has ever advised us how to make it that is not harmful" (Interviewee \#7).

D. Belief in diabetes only by eating sweet foods. Some women attributed high blood sugar levels to sugary foods: "Some patients are not aware that they think they should limit only sugar and the rest of the food is allowed to eat as much as they want" (Interviewee \#20).

E. Mental problems preventing diet (belief in uncontrollable appetite). Pre-diabetic women and the health care staff cited mental health problems such as depression and anxiety as one of the barriers to dieting. An interviewee said that "I cannot control myself and I eat too much when I am upset and angry about something" (Interviewee \#25).

F. Interest in eating ready-made and modern foods. One of the barriers to healthy nutrition that most women cited was the tendency of the younger generation to eat ready-to-eat, modern foods that did not include traditional foods: Interviewee \#41 stated: "Also, I should consume less fast food and fried foods in general. I cannot reduce the consumption of fast food at home because of my children's interest. I get used to eating myself".

3. Neutral perceptions. It is the third category in the data referring to perceptions and beliefs that do not prevent health behavior and do not need to be changed. It includes two subcategories: a greater tendency to follow a diet than to exercise, and cooking methods based on taste and smell (spicy foods).

A. A greater tendency to follow a diet than do physical activity. "Following a diet is easier than doing exercises" (Interviewee \#37).

B. Cooking methods based on taste and smell (spicy foods). "I often drink a lot of water because we eat spicy foods. I have a sign of excessive thirst, which is a sign of high blood sugar, which may be wrong" (Interviewee \#17).

\section{Enablers}

In this study, there are social forces that can be effective in increasing health behaviors or prevent them from occurring by creating barriers.

1. Positive enablers. There are social forces that can be effective in increasing health behaviors and facilitating the change of health behaviors and includes 4 subthemes: effective training of health care providers (physicians and experts), easy access to health care centers, media education (radio and TV), free services of the health centers (diabetes screening plan - tests, etc.).

A. Easy access to the health care center - effective training of the health care center. Pre-diabetic women described the presence of health centers near their place of residence and access to physicians and members of the health care team and their training as effective in informing them about the disease, how to control and prevent the progression of the disease and their becoming diabetic: "The physician told me to reduce my blood sugar level. I have to follow my diet. I do the same thing. I'm glad he didn't tell me to take medicine as my sister does" (Interviewee \#1).

B. Media education (radio and television). Prediabetic women valued the role of television as well as radio in raising their awareness of the disease and ways to control it: "Patients with pre-diabetes or pre-hypertension do not feel the need to listen to educational programs on mass media until they have the disease and become non-diabetic" (the health care center's physician).

C. Free health center services. "Some of the free services of the health center caused them to go to the health care center and get screened and find out they were pre-diabetic. They have to follow their diet" (the health care center's expert).

2. Negative enablers. There are social forces that can hinder health behaviors and included 5 sub-themes, lack of counseling facilities in the center, inadequate follow-up of pre-diabetics' conditions, inadequate education for pre-diabetics, inefficient referral system services, and insufficient family income. 
A. Lack of counseling facilities in the health care center. Pre-diabetic women and members of the health care team felt the lack of a nutritionist, sports, and mental health expert at the center. "We do not have an expert at the health care center" (the health care center's physician).

B. Inadequate follow-up of pre-diabetics' conditions. The participants complained about no filing and following up the pre-diabetics' health care services like diabetics': "It would have been much better if prediabetics' records had been recorded and filed, and like the diabetics who were followed up every three months, they were followed up as well" (the health care center's expert).

C. Inadequate education of pre-diabetics. Most prediabetic women reported that their health care center did not provide training classes on their disease at the health care center: "They should hold some courses for us on how to cook diet foods and what to eat to avoid diabetes" (Interviewee \#19).

\section{Neutral enablers}

A. Belief in the use of herbal medicines (traditional medicine) in controlling blood sugar levels. Many pre-diabetic women mentioned the experience of using herbal and home remedies. "I, with borderline diabetes, do not take medicine. But, I both follow my diet and eat fenugreek tea once a day" (Interviewee \#10).

B. Preparation of food by working together. Most of the pre-diabetic women mentioned the preparation of food with the help of other family members, as they felt it to be more motivating to make food. The Health care center's staff confirmed it as well: "When I'm going to cook, my two daughters help me. So, I cook more patiently" (the health care center's physician).

\section{Reinforcers}

They are knowledge, attitudes, perceptions, and behaviors of family, health care service providers, friends, and others who are significant to the predialectics, and the pre-diabetics follow them.

1.Positive reinforcers. Perceptions, beliefs, and behaviors of family, health care service providers, friends, and others who help change health behavior and include seven subcategories as follows:

A. Family members' (parents') encouragements

B. Other family members' encouragements

C. Friends' encouragements

D. Physicians' encouragements

E. Health staff's encouragements

D. Good communication and attitude of the center staff

E. Communication with individuals and families with diabetes

F. Positive behavioral experiences of dieting
G. Experience losing weight - lowering blood sugar levels- educing symptoms.

\section{Negative reinforcers}

Negative reinforcers are as follows:

A. High interest in consuming sugary foods and sweets

B. The idea of separating your food from the family food in case of a certain diet

C. Inability to follow a diet when eating with family members

D. The family members' insistence on neglecting the disease

E. The family members' mere advice to diet and their no accomplishment with the patient

F. The tendency of the family members, especially the youth (children), to consume fast food

G. The patient's no power in selecting and preparing food

H. Family lifestyle (low attention to self-care behaviors).

\section{Neutral reinforcers}

Family, health care service providers, friends, and others who do not affect health behaviors, including two sub-categories of:

A. Planning of food and preparation of a list with family members

B. Preparation of a shopping list of raw materials needed to prepare food with family members.

\section{Discussion}

Determinants of not following a proper diet, based on the experiences of pre-diabetic women and health care providers of their perceptions in three categories of positive perceptions, negative perceptions, and neutral perceptions were among the main of perceptual factors. They know the cause of their prediabetes or other chronic diseases, such as hyperlipidemia, hypertension, and obesity, which are related to poor diet. Pre-diabetic women adhered to their traditional diets but did not know enough about high-fiber, low-sugar foods, how to properly consume foods namely what they were allowed to eat, and how to properly eat a variety of healthy, traditional foods. Given that culture, eating habits, and nutritional literacy are the food patterns of any society [16]. The findings of this study also showed that inadequate awareness is an important factor in unhealthy nutrition in pre-diabetic women that leads to poor nutritional culture. It can also reduce motivation and the inability to follow a regular diet and plan properly. These findings are consistent with the results of Belio [17], Koshani [18], and Ramezani [19].

A review of the literature suggests that merely informing people is not effective and cannot change people's behaviors. As a result, in addition to awareness of attitudes, values, behavioral barriers, motivations, 
and goals of individuals to perform healthy behavioral patterns need to be considered [20]. In the present study, women who saw the effects of high blood sugar levels (such as leg ulcers, blindness, amputation, etc.) in their diabetic surroundings felt themselves at risk for those complications and followed a diet. But most of them were careless with their diet and also thought that if their disease was serious, their doctor would prescribe medicine.

In the study of Morowati et al., the perceived threat was higher in diabetic patients, especially those who observed the effects of high blood sugar levels in those around them than in pre-diabetic individuals [21]. In the present study, the main problem for women to follow a healthy and recommended diet was that they could not do it regularly and consistently because of being in situations such as parties, when they are stressed or angry, when they are eating with other family members when they are very hungry, etc. It refers to the Schwartzer's general self-efficacy, meaning that despite the difficulty of the behaviors and the existence of obstacles, they are confident in their ability to maintain that behavior [22].

The findings of this study showed that in the category "positive enablers", easy access to health care centers, the implementation of a free screening plan, and the effective training of health care team members had informed women about borderline diabetes (prediabetes). Therefore, in addition to health care providers in health care centers, having a mental health and nutrition counselor to increase awareness and control blood sugar levels can be very effective. However, the participants complained about the inefficiency of medical systems in providing health care services, lack of sufficient specialized staff in the health care center for counseling, especially nutritionists, inadequate education of pre-diabetics, and inconsistencies in their recommendations and confusion, inadequate follow-up pre-diabetics' conditions, failure to file their records, and imposition of additional costs on the pre-diabetics such as travel costs for health care if they were referred to places other than the health care centers and the need to travel long distances. They understood these inefficiencies as the reasons for not controlling their blood sugar levels. In Mousavizadeh et al. [23], the participants pointed to the inefficient treatment system and poor performance of the treatment team. Lack of training and counseling as well as the low quality of training or inconsistencies in the information presented in other studies have been mentioned as obstacles to the continuation of self-care. These results are consistent with the results of the present study [18, 24, 25].

As the findings show, the existence of sufficiently skilled educators, increase in the number of educa- tors, and the existence of adequate training programs to support the self-efficacy of pre-diabetic individuals to adhere to preventive behaviors, including diet and health care quality improvement using a patientcentered approach by health care providers can be effective in supporting pre-diabetics and removing these barriers.

Participants believed that media and advertising were effective in increasing their awareness of disease control and awareness of healthy eating behavior, but they did not need to listen to or watch such programs until they took the disease seriously and felt threatened. On the other hand, they pointed to the use of vague words by media experts and contradictions in their recommendations and teachings, especially inappropriate food advertisements, and as a result, the prevalence of unhealthy eating patterns in their children. Ramezani Tehrani pointed to the effect of mass media on food choices. These results are consistent with the results of the present study [26].

One of the negative reinforcers in the society that was seen as an obstacle to diet was the inadequacy of the existing services and educational programs in terms of culture, the lack of emphasis on nutrition education on traditional foods. To improve nutrition in a society, in addition to an adequate education program in that society, special measures must be taken by health policymakers following economic, social, and cultural conditions. The findings of previous studies confirmed these results of the present study $[27,28]$.

\section{Conclusion}

Since most women have good access to health care centers, it seems that the most appropriate and accessible way to promote diabetes prevention behaviors in pre-diabetics is to hold training classes with a health promotion approach implemented by health care providers. In these classes, in addition to increasing the awareness of pre-diabetics, creating a positive attitude towards health-promoting behaviors should be considered. Due to the effective role of social support in performing preventive behaviors and disease control, it is recommended to consider the role of families as a reinforcing factor in designing programs. Use the involvement of other literate family members in health promotion programs to improve health behaviors as well as information support is improtant. They can also help reinforce these behaviors in women and their families by providing appropriate educational content. Since the media has an important role in informing the public in the prevention and control of diseases, it is suggested that the impact of the media on raising public awareness in the prevention and control of 
diseases should be examined, barriers to the lack of effective medical and health care program should be studied, and those involved in the media should take action to remove these barriers.

\section{Limitations}

Since this study employed an exploratory sequential mixed methods design, the generalization of the qualitative results should be cautiously done. Also, because there was no similar study, it was not possible to discuss and compare extensively.

\section{Ethics approval}

The present research was approved with code IR.SSU.SPH.REC.1395.114 at the Ethics Committee from Yazd University of Medical Sciences.

\section{Conflicts of interest}

There are no conflicts of interest.

\section{REFERENCES}

1. Moradi A, Morovati HR, Teimourpour A, et al. Determine the prevalence of gestational diabetes in Ardakan and its related factors. MethodsX. 2019; 6: 409-416, doi: 10.1016/j.mex.2019.02.016, indexed in Pubmed: 30899677.

2. Olfatifar M, Karami M, Shokri P, et al. Prevalence of chronic complications and related risk factors of diabetes in patients referred to the diabetes center of hamedan Province. Scientific Journal of Hamedan Nursing and Midwifery Faculty. 2017; 25(2): 69-74, doi: 10.21859/nmj-25029

3. Moradi A, Alavi SM, Salimi M, et al. The effect of short message service (SMS) on knowledge and preventive behaviors of diabetic foot ulcer in patients with diabetes type 2. Diabetes Metab Syndr. 2019; 13(2): 1255-1260, doi: 10.1016/j.dsx.2019.01.051, indexed in Pubmed: 31336474.

4. Shabibi $P$, Mansourian $M$, Abedzadeh $M$, et al. The status of self-care behaviors in patients with type 2 diabetes in the city of Ilam in 2014. journal of ilam university of medical sciences. 2016; 24(2): 63-71, doi: 10.18869/acadpub.sjimu.24.2.63.

5. Jalilian H, Pezeshki MZ, Torkzadeh L, et al. Health care seeking behaviors in type 2 diabetic patients in East Azerbaijan. Clinical Diabetology. 2020; 8(6): 292-302, doi: 10.5603/ dk.2019.0031.

6. Nikbina M, Mameneh $M$, Bakaeian $M$, et al. Effectiveness of nutrition education and counseling on metabolic control parameters of diabetes mellitus type 2 patients in primary health care centers. Clinical Diabetology. 2020; 9(5): 293-299, doi: 10.5603/ DK.2020.0030.

7. Hafez Griauzde D, Saslow L, Patterson K, et al. Mixed methods pilot study of a low-carbohydrate diabetes prevention programme among adults with pre-diabetes in the USA. BMJ Open. 2020; 10(1): e033397, doi: 10.1136/bmjopen-2019-033397, indexed in Pubmed: 31969366.

8. Hu FB. Globalization of diabetes: the role of diet, lifestyle, and genes. Diabetes Care. 2011; 34(6): 1249-1257, doi: 10.2337/ dc11-0442, indexed in Pubmed: 21617109.

9. Peyman N, Ezzati Ra, Taghipour A, et al. Effectiveness of education by PEN-3 cultural model on improve feeding behaviors in adolescent girls with obesity and over weight in Razan city (Hamadan)-2010. Horizon Med Sci. 2013; 18(5): 254-260.

10. Shahbazi $H$, Ghofranipour $F$, Amiri $P$, et al. Factors affecting selfcare performance in adolescents with type i diabetes according to the PEN-3 cultural model. Int J Endocrinol Metab. 2018; 16(4): e62582, doi: 10.5812/ijem.62582, indexed in Pubmed: 30464772.

11. Moghaddam ES, Shahnazi H, Hassanzadeh A. Predictive power of PEN-3 model constructs in breast cancer screening behaviors among teachers: a cross- sectional study in Central Iran. Eur J Breast Health. 2019; 15(2): 105-110, doi: 10.5152/ ejbh.2019.4417, indexed in Pubmed: 31001612.

12. Yick A, Oomen Early J. Using the PEN 3 model to plan culturally competent domestic violence intervention and prevention services in Chinese American and immigrant communities. Health Education. 2009; 109(2): 125-139, doi: 10.1108/09654280910936585.

13. , et al Bartholomew LK, Parcel GS, Kok G, et al. Planning health promotion programs: anintervention mapping approach. JosseyBass 2006.

14. Graneheim UH, Lundman B. Qualitative content analysis in nursing research: concepts, procedures and measures to achieve trustworthiness. Nurse Educ Today. 2004; 24(2): 105-112, doi: 10.1016/j.nedt.2003.10.001, indexed in Pubmed: 14769454.

15. Sirati Ni, Ebadi A, et al. Fallahi Khoshknab M, Consequences of living with Posttraumatic Stress Disorder: A Qualitative Study. Journal of Qualitative Research in Health Sciences. 2012; 1(2): 92-101.

16. Hosseyni Esfahani F, Jazayeri A, Mirmiran P, et al. Dietary patterns and their association with socio-demographic and lifestyle factors among Thehrani adults: Tehran Lipid and Glucose Study. Journal of School of Public Health and Institute of Public Health Research. 2008; 6(1): 23-36.

17. BeLue R, Diaw M, Ndao F, et al. A cultural lens to understanding daily experiences with type 2 diabetes self-management among clinic patients in M'bour, Senegal. Int Q Community Health Educ. 2012; 33(4): 329-347, doi: 10.2190/IQ.33.4.b, indexed in Pubmed: 24044925.

18. Keshani P, Farvid MS. Perceived benefits and barriers regarding high fiber food intake in type 2 diabetes patients-a qualitative study. Iranian Journal of Nutrition Sciences \& Food Technology. 2012; 7(1): 11-22.

19. Ramezani Te, Amiri P, Paikari N, et al. Metabolism. Women's perception regarding to healthy nutrition inhibitors: a qualitative research in tehran lipid and glucose study (TLGS). Iranian Journal of Endocrinology and Metabolism. 2011; 13(1): 48-57.

20. Sabzmakan L, Jafarabadi MA, Nikpajouh A, et al. Determinants of physical activity among patients with cardiovascular metabolic risk factors based on the educational factors of PRECEDE model: a mixed method study. ranian Journal of Health Education and Health Promotion. 2016; 4(1): 5-19, doi: 10.18869/acadpub. ihepsaj.4.1.5.

21. Sabzmakan L, Mazloomy Mahmoodabad S, Ali Morowatisharifabad M, et al. Patients, Experiences with Cardiovascular Disease Risk Factors and Healthcare Providers of Determinants of the Nutritional Behavior: A Qualitative Directed Content Analysis. Iranian Journal of Endocrinology and Metabolism. 2013; 15(3): 292-302.

22. Schwarzer R. Modeling health behavior change: how to predict and modify the adoption and maintenance of health behaviors. Applied Psychology. 2008; 57(1): 1-29, doi: 10.1111/j.14640597.2007.00325.x.

23. Mousavizadeh S, Ashktorab T, Ahmadi F, et al. Evaluation of barriers to adherence to therapy in patients with diabetes. J Diabetes Nurs. 2016; 4(3): 94-108.

24. Lewis CP, Newell JN. Patients' perspectives of care for type 2 diabetes in Bangladesh -a qualitative study. BMC Public Health. 2014; 14: 737, doi: 10.1186/1471-2458-14-737, indexed in Pubmed: 25047797.

25. Zalak K, Kazemi Haki B, Matlabi H. Obstacles to self-care from the viewpoint of type II diabetic patients and guidelines to remove them. Jorjani Biomed J. 2012; 0(1): 30-37.

26. Farahmand $M$, Tehrani FR, Amiri $P$, et al. Barriers to healthy nutrition: perceptions and experiences of Iranian women. BMC Public Health. 2012; 12: 1064, doi: 10.1186/1471-2458-12-1064, indexed in Pubmed: 23227832. 
27. James DCS. Factors influencing food choices, dietary intake, and nutrition-related attitudes among African Americans: application of a culturally sensitive model. Ethn Health. 2004; 9(4): 349-367, doi: 10.1080/1355785042000285375, indexed in Pubmed: 15570680 .
28. Shea $S$, Stein AD, Basch $C E$, et al. Independent associations of educational attainment and ethnicity with behavioral risk factors for cardiovascular disease. Am J Epidemiol. 1991; 134(6): 567-582, doi: 10.1093/oxfordjournals.aje.a116130, indexed in Pubmed: 1951262. 